\title{
Paired States in the Even Integer Quantum Hall Effect
}

\author{
A. M. Tikofsky and S. A. Kivelsont \\ Institute for Theoretical Physics, University of California \\ Santa Barbara, CA 93106
}

(June 12, 1995)

\begin{abstract}
We argue that a new type of quantum Hall state requiring non-perturbative Landau level mixing arises at low electron density. In these states, up and down spin electrons pair to form spinless bosons that condense into a bosonic quantum Hall state. We describe a wavefunction for a paired quantum Hall state at $\nu=2$ and argue that it is stabilized by a BCS instability arising in flux attachment calculations. Based on this state, we derive a new global phase diagram for the integral quantum Hall effect with spin. Additional experimental implications are discussed.
\end{abstract}

PACS numbers: 73.40.Hm, 74.20.Kk

It is common to distinguish two different types of quantum Hall effects: the integer and the fractional [1]. When an integer number of Landau levels are filled and the kinetic energy cost for occupying higher Landau levels is large enough, the occupation of higher Landau levels encouraged by many-body interactions can be treated perturbatively. In the fractional quantum Hall effect, manybody interactions stabilize a ground state with a gap to excitations even though only a fraction of a Landau level is occupied. It is assumed that only a single Landau level is partially occupied, because of the large kinetic energy cost for occupying higher Landau levels, and that this state is adiabatically connected to the state with higher Landau level occupation. We will argue that this traditional picture is incomplete. When the energy gap between Landau levels is small enough, there is a new even integer quantum Hall effect that is distinct from the old one, and whose stability relies on the existence of manyparticle interactions and higher Landau level occupation.

This work is motivated by the fact that the topology of the experimental phase diagram is inconsistent with an integral quantum Hall effect insensitive to manyparticle interactions [2 [1. Of particular interest is the existence of a direct second order phase transition from $\nu=2$ to $\nu=0$ with the usual quantum Hall critical exponents (See Fig. 1; $\nu$ is defined as the value of the Hall conductivity $h \sigma_{x y} / e^{2}$ in fundamental units). In the non-interacting picture, the system is required to pass through an intermediate $\nu=1$ phase in the transition from $\nu=2$ to $\nu=0$ [1]. However, there is no experimental evidence for even a very thin region separating $\nu=2$ and $\nu=0$. We therefore postulate the existence of a new quantum Hall state, which we call the $2 b$ state, that is distinct from the non-interacting spin-unresolved $2 a$ state. The transition from the $2 a$ to the $2 b$ state would be first order in the absence of disorder and is probably second order in the presence of disorder.

The global phase diagram implies that there are direct transitions between even-integer quantum Hall states in low magnetic fields. In fact, at low enough magnetic fields, the odd-integer quantum Hall effect is known to disappear [2]. In addition, the transition directly from $\nu=0$ to $\nu=2$ and back to $\nu=0$ at low magnetic fields, shown in the phase diagram, has also been observed experimentally [顺].

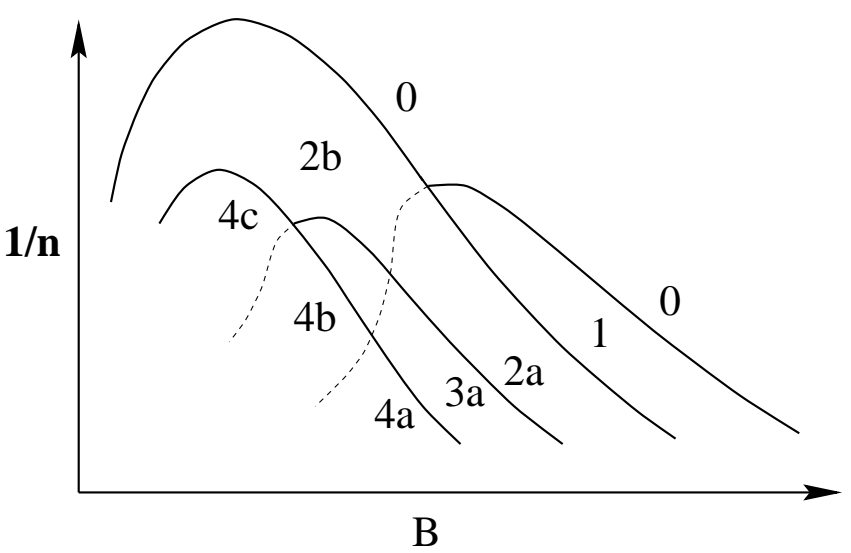

FIG. 1. The new global phase diagram for the Integral Quantum Hall Effect. Transitions between the same quantized hall conductance are indicated by dashed lines.

The essential idea behind the $2 b$ quantum Hall state is that up and down spin electrons pair to form a spinless boson that condenses into a bosonic many-body fractional quantum Hall state. It is clear this state must have significant occupation of higher Landau levels because the conventional spin unpolarized $2 a$ state is the unique state at $\nu=2$ in the lowest Landau level. For purposes of illustration, we describe a spin-singlet many electron wavefunction [5] for the $2 b$ state given by

$$
\Phi=\prod_{k<l, \sigma}\left(z_{k}^{\sigma}-z_{l}^{\sigma}\right) \operatorname{Per}\left[F\left(\left|z_{i}-z_{j}\right|\right)\right] e^{-\sum_{i}\left|z_{i}\right|^{2} / 4 l^{2}}
$$

where $z_{i}$ is the coordinate of the $\mathrm{i}^{\text {th }}$ electron, $z_{k}^{\sigma}$ is the coordinate of the $\mathrm{k}^{t h}$ spin $\sigma$ electron, $l$ is the magnetic length, and Per denotes the Permanent of the symmetric matrix whose $(i, j)^{t h}$ component is $F\left(\left|z_{i}-z_{j}\right|\right)$. Given an order $\mathrm{N}$ matrix $\mathrm{M}_{i j}$, the permanent $\operatorname{Per}(M)=H_{1}(M)$ where $H_{\alpha}(M)=\sum_{P}(\alpha)^{S\{P\}} \prod_{a b} M_{a b}$, the product is 
over the $N / 2$ pairs $a b$, the sum is over the possible permutations of these pairs, and $S\{P\}$ is -1 or +1 if the permutation $P$ is odd or even, respectively [6.7]. The Pfaffian of a matrix $\mathrm{M}$ is $\operatorname{Pf}(M)=H_{-1}(M)$.

The behavior of the $2 b$ state described in Eq. (11) is governed by the function $F$. For $F=1, \Phi$ is the conventional $2 a$ state. On the other hand, if we take $F$ to be a delta function then we can define a pair coordinate and $\Phi$ vanishes as the second power of the pair coordinate as two pairs approach each other. This wavefunction is therefore equivalent to a $\nu^{\prime}=1 / 2$ Laughlin state for bosonic pairs of charge $e^{*}=2 e$. Its Hall conductivity is $\sigma_{x y}=\left(e^{*}\right)^{2} \nu^{\prime} / h=2 e^{2} / h$. If $F$ is short-ranged then it can be thought of as a pair wavefunction and its effective size is the coherence length. For distances much longer than this coherence length, $F$ acts like a delta-function and $\Phi$ appears to be a $\nu^{\prime}=1 / 2$ state. The $2 a$ to $2 b$ transition is thus characterized by the divergence of this coherence length.

At first, the existence of a pairing state stabilized by repulsive Coulomb interactions seems antintuitive. Moreover, because $\Phi$ in Eq. (11) is constructed by occupying higher Landau levels, we lose kinetic energy that must be compensated by a gain in interaction energy. However, we only lose interaction energy among the two electrons in a pair; we gain repulsive energy from pairing because the $2 b$ state acts like a Laughlin $\nu^{\prime}=1 / 2$ state for pairs. As a first approximation, let us ignore the energy cost in the $2 b$ state for forming tightly bound pairs and calculate the interaction energy for all electrons not in the same pair. This is simply the Coulomb energy of a $\nu^{\prime}=1 / 2$ state of spinless charge $e^{*}=2 e$ bosons. Laughlin's interpolation formula for $E_{m}$ [1] , the Coulomb energy of a projected $\nu=1 / m$ state, gives an energy per electron for the $2 b$ state of $E_{2 b}^{*}=-.49\left(e^{*}\right)^{2} / \epsilon l^{*}=-1.39 e^{2} / \epsilon l$. In contrast, the energy of the $2 a$ state is $E_{2 a}=-\sqrt{\pi / 8} e^{2} / \epsilon l$. Forming a paired state has yielded an energy gain per pair of $2\left(E_{2 a}-E_{2 b}^{*}\right)=1.53 e^{2} / \epsilon l$ which must more than compensate the Coulomb and kinetic energy lost by pairing if the $2 b$ state is to be energetically stable. This requirement is made easier by the softening of the Coulomb repulsion at short distances due to the finite thickness of the electron gas [1],6].

As the magnetic field decreases, Coulomb energy becomes more important and the Coulomb energy gain of a $2 b$ state with respect to a $2 a$ state can more easily outweigh the loss of kinetic energy. To get a feeling for the magnitude of the magnetic field at which such a transition could occur, we study the properties of the $2 a$ state perturbatively in powers of the ratio of the interaction energy to the kinetic energy,

$$
y=\frac{e^{2} / \epsilon l}{\hbar \omega_{c}}=l / a^{*}=\sqrt{\frac{B^{*}}{B}},
$$

where $a^{*}=\epsilon \hbar^{2} / m^{*}$ is the effective Bohr radius and $m^{*}$ is the band effective mass. The $2 a$ state is exact in the infinite magnetic field or $y=0$ limit and is perturbatively stable for sufficiently small $y$. Failure of perturbation theory, which we take to signal a transition, would occur at a critical value of $y_{c} \sim 1$. For concreteness, we take $y_{c}=2.3$, the value at which the second order perturbative expression for the gap of the $2 a$ state vanishes [8]. Given material parameters for $G a A s-A l_{x} G a_{1-x} A s$ of $a^{*}=100 \AA$ and $B^{*}=6.6 T$, this value of $y_{c}$ corresponds to a magnetic field of $B=B^{*} / y_{c}^{2}=1.3 T$ which is in the same range as $.5 T$, the experimental magnetic field at which the $\nu=0,1$, and 2 quantum Hall states coincide (see Fig. 1) [2].

BCS Instability: We will employ the technique of flux attachment to show that a spin degenerate electron gas at $\nu=2$ has the pairing instability necessary to stabilize the $2 b$ state $[9,10]$. This procedure has the advantage that it implicitly describes wavefunctions not projected into the lowest Landau level and provides a motivation, other than energetics, for constructing a paired wavefunction. We choose as our basis a set of quasiparticles that are defined as an electron attached to one unit of spin-relative flux. This construction is accomplished by coupling $j_{\mu}^{\sigma}$, the $\mu^{\text {th }}$ component of the current of spin $\sigma$ electrons, to the gauge field $a_{\sigma}^{\mu}$, and requiring that

$$
\nabla \times \mathbf{a}_{\sigma}(\mathbf{r})=2 \pi \rho_{-\sigma}\left(\mathbf{r}^{\prime}\right)
$$

where $\rho_{\sigma}(\mathbf{r})$ denotes the density of spin $\sigma$ electrons. This flux attachment makes unlike spins relative bosons and keeps like spins relative fermions. The spin $\sigma$ quasiparticles see a mean magnetic field

$$
B_{e f f, \sigma}=\nabla \times\left(\mathbf{A}-<\mathbf{a}_{\sigma}>\right),
$$

which is zero since a $\nu=2$ spin-unpolarized state satisfies $\nabla \times \mathbf{A}=2 \pi<\rho_{\sigma}(\mathbf{r})>$. At the mean-field level, the quasiparticles form a spin-degenerate Fermi sea because like spins are non-interacting relative fermions in zero magnetic field. We can calculate the effect of fluctuation corrections on both the system's linear-response function and the dressed interparticle interaction [11]. First of all, we define the linear-response function $\Pi$ by the statement that a spin-dependent gauge field $A_{\text {ext }}^{\sigma^{\prime}}$ of frequency $\omega$ and wavevector $\mathbf{q}$ induces a current of spin $\sigma$ quasiparticles

$$
J_{\mu}^{\sigma}(\omega, \mathbf{q})=\Pi_{\mu \nu}^{\sigma, \sigma^{\prime}}(\omega, \mathbf{q}) A_{e x t, \nu}^{\sigma^{\prime}}(\omega, \mathbf{q})
$$

where $\mu, \nu$ take on the values $(0, \mathrm{x}, \mathrm{y})$. We follow the standard convention of working in Coulomb gauge so that $\mathbf{q} \cdot \mathbf{A}^{\sigma}=\mathbf{q} \cdot \mathbf{a}^{\sigma}=0$. The linear-response kernel of the system can therefore be described by using only the $4 \times 4$ submatrix $\Pi_{\alpha \beta}^{\sigma, \sigma^{\prime}}$ in which the subindices $\alpha$ and $\beta$ take on the values 0 and 1 corresponding to the time and transverse component of a vector, respectively. In this notation, the response function of the mean-field degenerate Fermi sea is

$$
K_{\alpha \beta}^{\sigma, \sigma^{\prime}}(q, \omega)=\delta^{\sigma, \sigma^{\prime}} \delta_{\alpha \beta} P_{\alpha \alpha}(q, \omega)
$$

while the bare momentum space interaction is 


$$
V_{\alpha \beta}^{\sigma, \sigma^{\prime}}(q, \omega)=\frac{2 \pi e^{2}}{\epsilon q} \delta_{\alpha, o} \delta_{\beta, o}+\frac{2 \pi i}{q} \epsilon_{\alpha \beta}\left(1-\delta^{\sigma, \sigma^{\prime}}\right) .
$$

We have assumed a spin-independent Coulomb interaction between electrons. In the RPA [9, 11], the screened momentum space interaction $D$ and response kernel $\Pi$ satisfy the matrix equations

$$
D=V+V K D \text {, and } \quad \Pi=K+K V \Pi \text {. }
$$

We now use this flux attachment formalism to show that the system has an instability towards singlet BCS pairing. Because we are interested in spin-singlet pairing and have transmuted the particles into relative bosons, the pairing must be odd parity. The BCS instability can be shown to be strongest in the p-wave channel, the lowest allowed angular momentum channel. The screened interaction $D$, given in Eq. (8), yields the BCS pair interaction in the singlet channel

$$
\begin{aligned}
V\left(\mathbf{k}, \mathbf{k}^{\prime}, \omega\right) & =D_{00}^{\uparrow \downarrow}(q, \omega)+\left(\mathbf{k} \times \mathbf{k}^{\prime}\right) D_{01}^{\uparrow \downarrow}(q, \omega) / q \\
& +\left|\mathbf{k} \times \mathbf{k}^{\prime}\right|^{2} D_{11}^{\uparrow \downarrow}(q, \omega) / q^{2} .
\end{aligned}
$$

where $q=\left|\mathbf{k}-\mathbf{k}^{\prime}\right||6,12|$. We assume a gap function with odd $m$-wave pairing, $\Delta(\mathbf{k})=|\Delta(k)| e^{-i m \phi_{\mathbf{k}}}$. Because $D_{01}^{\sigma, \sigma^{\prime}}$ is pure imaginary and $V\left(\mathbf{k}, \mathbf{k}^{\prime}, \omega\right)$ thus has a non-vanishing imaginary component, the pairing instability in the BCS gap equation, for a fixed $|m|$, is strongest for $m<0$. However, the dominant attraction in all angular momentum channels comes from $D_{11}^{\uparrow \downarrow}(q, \omega)$ [12,13].

A spin-singlet wavefunction corresponding to the flux attachment calculations is given by

$$
\Phi^{\prime}=\prod_{k, l} \frac{1}{\left(z_{k}^{\uparrow}-z_{l}^{\downarrow}\right)^{*}} \operatorname{Pf}\left[\left(z_{i}-z_{j}\right) G_{i j}\right] e^{-\sum_{i}\left|z_{i}\right|^{2} / 4 l^{2}}
$$

instead of Eq. (11) where $G_{i j}=G\left(\left|z_{i}-z_{j}\right|\right)$ and the Pfaffian is defined in conjunction with Eq. (1). The prefactor in Eq. (10) implements the singular gauge transformation described in Eq. (3) and can be shown to make $\Phi^{\prime}$ into a spin-singlet wavefunction. (If we had chosen to implement the gauge transformation via the prefactor $\prod_{k, l}\left(z_{k}^{\uparrow}-z_{l}^{\downarrow}\right)$ then $\Phi^{\prime}$ would not be a spin-singlet.) In contrast to the gauge theory description of $\Phi^{\prime}$, a similar description of $\Phi$, given in Eq. (1), would transmute the electrons into bosonic quasiparticles. Therefore, a pairing mechanism describing $\Phi$ would have to bind two charge $e$ bosons into a charge $2 e$ boson [14. Nevertheless, we believe that both $\Phi$ in Eq. (11) and $\Phi^{\prime}$ describe the same physics in the thermodynamic limit.

The physical behavior of the paired state is most easily expressed in terms of the gauge field and current combinations [15]:

$$
a^{ \pm}=\frac{1}{2}\left(a^{\uparrow} \pm a^{\downarrow}\right), \quad j_{\mu}^{ \pm}=j_{\mu}^{\uparrow} \pm j_{\mu}^{\downarrow} .
$$

The $j^{+}$current only couples to the $a^{+}$gauge field while the $j^{-}$current only couples to the $a^{-}$gauge field. A singlet paired state has a gap $\Delta_{s}$ for single particle excitations in both the $j^{+}$and $j^{-}$channels. In addition, the $j^{+}$ channel has a gapless Goldstone mode whose spectrum is given by the poles of $a^{+}$'s propagator. While fluctuations of the statistical gauge field $a$ do not affect the low-energy spectrum of the $j^{-}$channel [16], they gap the Goldstone mode of the $j^{+}$channel and give it a quantized $\nu=2$ Hall effect [9 11]. Both of these effects are apparent in the screened response functions $\Pi^{ \pm}=2\left(\Pi^{\uparrow \uparrow} \pm \Pi^{\uparrow \downarrow}\right)$ where $\Pi$ is defined in Eq. (8) and spin-symmetry assumed.

Implications: The $2 b$ state has a number of different quasiparticle excitations. Because the paired state describes spinless bosons at filling fraction $\nu^{\prime}=1 / 2$, we can construct the usual quantum Hall quasiparticles with charge $\pm e^{*} \nu^{\prime}= \pm e$ and statistics $\pm \pi \nu^{\prime}= \pm \pi / 2$. We refer to these charge 1 spinless semions as holons. When we bind a single electron to a holon, we make a charge 0 spin $1 / 2$ semion which we refer to as a spinon. In addition, electrons are also quasiparticle excitations and may be viewed as a bound spinon-holon pair.

Let us now construct quantum Hall hierarchies by using the $2 b$ paired state as a parent state. Because electrons are well-defined quasiparticles, one can imagine constructing conventional integral quantum Hall states of up and down spin electrons on top of the parent $2 b$ state. These are called $n b$ states in the figure. On the other hand, we can pair up and down spin electrons into a new boson and form a $\nu^{\prime}=1 / 2$ bosonic quantum Hall states of these new pairs. The $4 c$ state shown in Fig. 1 is an example of such a state. We can construct yet another hierarchy of paired spin-unpolarized states based on the condensation of holons at filling fractions $\nu=4 \nu^{\prime}=8 p /(4 p+1)$ where $p$ is an integer [1].

In analogy to the $2 b$ state, we have constructed other singlet paired states at filling fraction $\nu=2 /(2 p+1)$ where $\mathrm{p}$ is an integer. These states correspond to bosonic quantum Hall states of charge $2 e$ bosons at filling fraction $\nu^{\prime}=1 / 2(2 p+1)$. If we try to construct triplet paired states, spin-rotation symmetry requires them to be spin-polarized. However, there is no BCS instability for spin-polarized states; in fact, the $\omega=0$ BCS interaction is repulsive in all angular momentum channels [12]. Therefore, these states are unlikely to exist in nature unless spin symmetry is broken. In fact, there is evidence for triplet pairing in bilayer systems which do not have pseudospin-rotation (layer index) symmetry [17].

Let us now address the nature of the transitions between the various states shown in Fig. 1, in the presence of dirt. It is well established that the transitions between daughter and parent quantum Hall states are second order and are all similar to each other. These transitions can be viewed as the quantum percolation transition of a single edge state with the selection rule 18] that only one state can undergo this transition at a time. In this language, the $2 a$ state has 2 edge modes, one composed of each spin polarization, and the $2 a$ to 1 transition is associated with the percolation of the down spin edge state. Alternatively, for small Zeeman energy, the $2 a$ state can be viewed as having a charged holon edge mode and a neutral spinon edge mode. The transition from $2 a$ to $2 b$ 
can therefore be viewed as spinon percolation. We postulate that this transition is similar to the $2 a$ to 1 transition but that the critical behavior is visible in the spin degrees of freedom and that the system is a spin-metal at the critical point. In addition, we identify the tetracritical point in Fig. 1 as the intersection of the phase boundaries associated with the two order parameters: the Girvin-MacDonald order parameter defined by attaching one unit of $\mathbf{a}^{\uparrow}$ flux to the up spin electron creation operator is nonzero in both the 1 and $2 a$ phases; the order parameter defined by attaching one unit of $\left(\mathbf{a}^{\uparrow}+\mathbf{a}^{\downarrow}\right) / 2$ flux to the charge $2 e$ pair creation operator is nonzero in both the $2 a$ and $2 b$ phases [1].

Other Experimental Implications: It has been conjectured that there is a universal value for the resistance at quantum hall phase transitions [18. Recent experiments have in fact found universal values for the resistance at quantum hall liquid to insulator transitions [2 [21]. According to the pairing theory, the transition between $\nu=2$ and $\nu=0$ corresponds to a $\nu^{\prime}=1 / m=1 / 2$ quantum hall liquid to insulator transition of charge $e^{*}=2 e$ quasiparticle with the corresponding universal resistances [18: $\rho_{x x}=h /\left(e^{*}\right)^{2}=h / 4 e^{2}$ and $\rho_{x y}=m h /\left(e^{*}\right)^{2}=h / 2 e^{2}$. This prediction is consistent with the observed values for the resistances in the experiments of Wong et. al. [2]. (It is important to note that there are experiments at higher magnetic fields that find different resistances at the $\nu=2$ to insulator phase transition or a different critical exponent [4]. This discrepancy is not yet understood.)

Our theory predicts a spin gap $\Delta_{s}$ associated with pairing. In the presence of dirt, this may become a pseudogap. Nonetheless, this gap should be experimentally observable by studying the density of free spins as a function of $\delta B=B-\pi \rho$ 22]. The density of free spins should grow linearly with $|\delta B|$ in the $2 a$ state while it should be strongly suppressed in the $2 b$ state for temperatures below $\Delta_{s}$, even for $\delta B \neq 0$. In addition, the Zeeman splitting should act as a pair breaker and decrease the spin gap $\Delta_{s}$. We therefore expect the $2 b$ state to be destroyed when the Zeeman energy exceeds the spin gap $\Delta_{s}$. Indeed, the triple point, where $\nu=0,1$, and 2 coexist in Fig. 1, is pushed to lower fields as the Zeeman energy is increased by tilting the magnetic field [2],

The point contact conductance of the $2 b$ state will be very different than that of a $2 a$ state. The $2 a$ state has 2 Fermi-liquid edge modes with nonzero conductance at zero temperature [19]. In contrast, the $2 b$ state has only a single $\nu^{\prime}=1 / 2$ edge mode of charge $e^{*}$ carriers. For sufficiently low temperatures, the point contact tunneling will be non-Fermi-liquid like and the conductance will vanish as $T^{2}$ at very low temperatures 19,20]. In addition, the scaling function for the resonance line shape, in the limit of small temperature, is known exactly for $\nu^{\prime}=1 / 2$. In the limit of strong backscattering (large gate voltage), the conductance is dominated by single electron tunneling and hence is thermally activated because of the gap $\Delta_{s}$ to single particle excitations. This should provide an experimental measure of $\Delta_{s}$.

In future work, we will explore in detail the energetic properties of the proposed paired wavefunctions for finite-size systems.

We are grateful to $\mathrm{H}$. W. Jiang for sharing his results with us prior to publication. We have also benefited from many useful conversations with N. E. Bonesteel, A. H. Castro-Neto, M.P.A. Fisher, D. Morse, S. L. Sondhi, and A. Zee. This work has been supported by the National Science Foundation under grant PHY94-07194 at the Institute for Theoretical Physics and grant DMR93-12606 at UCLA.

* Permanent Address: Dept. of Physics, University of California, Los Angeles, CA 90024.

[1] R. Prange and S. M. Girvin, The Quantum Hall Effect (Springer-Verlag, New York, 1987).

[2] L. W. Wong et. al., Phys. Rev. B52, 18033 (1995); H.W. Jiang, unpublished.

[3] H. W. Jiang et. al., Phys. Rev. Lett. 71, 1439 (1993);

[4] T. Wang et. al., Phys. Rev. Lett. 72, 709 (1994); R. J. F. Hughes et. al., J. Physics: Cond. Mat. 6, 4763 (1994).

[5] I. Herbut, Phys. Rev. B46, 15582 (1992).

[6] M. Greiter et. al., Nuc. Phys. B374, 567 (1992).

[7] G. Moore and N. Read, Nuc. Phys. B360, 362 (1991).

[8] S. L. Sondhi et. al., Phys. Rev. B47, 16419 (1993).

[9] S. C. Zhang et. al., Phys. Rev. Lett. 62, 82 (1989); A. L. Fetter et. al., Phys. Rev. B39, 9679 (1989); A. Lopez and E. Fradkin, Phys. Rev. B47, 7080 (1993).

[10] D. H. Lee and C. L. Kane, Phys. Rev. Lett. 64, 1313 (1990).

[11] B. I. Halperin et. al., Phys. Rev. B47, 7312 (1993).

[12] N. E. Bonesteel, Phys. Rev. B48, 11484 (1993); N. E. Bonesteel, in preparation.

[13] The details of this calculation are discussed in ref. 12 as well as A. M. Tikofsky, unpublished.

[14] M. J. Rice and Y. R. Wang, Phys. Rev. B37, 5893 (1988).

[15] X. G. Wen and A. Zee, Phys. Rev. B47, 2265 (1993).

[16] S. C. Zhang et. al., Phys. Rev. Lett. 69, 1252 (1992).

[17] M. Greiter et. al., Phys. Rev. B 46, 9586 (1992).

[18] S. Kivelson et. al., Phys. Rev. B46, 2223 (1992).

[19] X. G. Wen, Phys Rev. B44, 5708 (1991).

[20] C. L. Kane and M. P. A. Fisher, Phys. Rev. B46, 15233 (1992); ibid., Phys. Rev. B51, 13449 (1995).

[21] D. Shahar et. al., Phys. Rev. Lett. 74, 4511 (1995).

[22] S. E. Barrett et. al., Phys. Rev. Lett. 74, 5112 (1995). 